\title{
CEACAM proteins: emerging biomarkers and targets in metastatic cancer
}

N. Beauchemin and A. Arabzadeh

Goodman Cancer Research Centre and Depts. of Biochemistry, Medicine and Oncology; McGill University, Montreal, Canada.

Background: Discovery of the Carcinoembryonic Antigen (CEA) as a biomarker for colorectal cancer some 50 years ago became the first step in the identification of a much larger family of 12 Carcinoembryonic Antigen-related Cell Adhesion Molecules (CEACAMs). These proteins form a large cluster of immunoglobulin-related proteins on chr. 19 with some being cell-surface attached and others secreted. They exhibit surprisingly diverse functions in cell adhesion, intra-and inter-cellular signalling and during complex biological processes, such as cancer progression, inflammation, angiogenesis and metastasis.

AIMS: In this review, we will discuss research developments of the last 10 years involving three of the most prominent family members, CEACAM1, CEACAM5
(CEA) and CEACAM6 and their involvement in melanoma, lung, colorectal and pancreatic cancers.

Methods: Development by a large number of CEACAM-oriented laboratories of proper molecular and biochemical tools and mouse models (L-SACC transgenic and Ceacam1-/- mouse models) has enabled bi-directional translation of the CEACAM network biology.

Results: CEACAM1, CEACAM5 (CEA) and CEACAM6 cooperate with cell surface receptors, integrins, cytoskeletal proteins and extracellular matrix proteins to influence the outcome of proliferation, apoptosis, progression, angiogenesis and immune responses in melanoma, lung, colorectal and pancreatic cancers.

Conclusion: These proteins represent valid clinical biomarkers and promising cancer therapeutic targets. These are fascinating successors to the old tumor marker-CEA. 